\title{
Overexpression of PBK/TOPK Relates to Poor Prognosis of Patients With Breast Cancer: A Retrospective Analysis
}

\section{Liang Qiao}

Zhongshan Hospital Affiliated to Dalian University

Jinling Ba

Zhongshan Hospital Affiliated to Dalian University

Jiping Xie

Zhongshan Hospital Affiliated to Dalian University

Ruiping Zhu

Zhongshan Hospital Affiliated to Dalian University

Yi Wan

Zhongshan Hospital Affiliated to Dalian University

\section{Min Zhang}

Zhongshan Hospital Affiliated to Dalian University

\section{Zeyu Jin}

Zhongshan Hospital Affiliated to Dalian University

\section{Zicheng Guo}

Zhongshan Hospital Affiliated to Dalian University

Jiaxuan Yu

Zhongshan Hospital Affiliated to Dalian University

\section{Sijing Chen}

Zhongshan Hospital Affiliated to Dalian University

Yongqiang Yao ( $\nabla$ dlyyq0411@163.com )

Zhongshan Hospital Affiliated to Dalian University

\section{Research Article}

Keywords: PBK/TOPK, over-expressed, breast cancer, prognosis

Posted Date: January 14th, 2022

DOI: https://doi.org/10.21203/rs.3.rs-1250525/v1 
License: (c) (i) This work is licensed under a Creative Commons Attribution 4.0 International License. Read Full License 


\section{Abstract}

Background: PDZ-binding kinase/T lymphokine-activated killer cell-derived protein kinase (PBK/TOPK) is a potential prognostic indicator for patients with breast cancer. The objective of the present study was to explore the relationship between PBK/TOPK expression and clinicopathological indicators as well as the survival of patients with breast cancer.

Methods: Immunohistochemical staining was used to detect the expression of PBK/TOPK in 202 cases of breast cancer tissues. The relationship between PBK/TOPK and clinicopathological parameters was evaluated using Spearman's rank-order correlation. The difference in PBK/TOPK expression among different molecular types was analyzed with the chi square test. Kaplan-Meier analysis was used to create a survival curve and the log rank test was used to analyze the overall survival (OS) and diseasefree survival (DFS). Prognostic correlation was assessed using univariate and multivariate Cox regression analyses.

Results: Among 202 breast cancer samples, PBK/TOPK was expressed ('+' and '++') in 182 samples (90.1\%). In addition, the histological grade, TNM stages, lymph node metastasis, estrogen receptor (ER), progesterone receptor (PR), human epidermal growth factor receptor 2 (HER-2), and Ki-67 were positively associated with PBK/TOPK expression. With regard to the molecular type, the expression of PBK/TOPK is different. The expression level of PBK/TOPK was negatively correlated with both the OS and DFS of breast cancer patients. The difference in the above results is meaningful $(P<0.05)$.

Conclusions: PBK/TOPK is over-expressed in breast cancer and the expression is closely related to the clinicopathological characteristics of the disease. Breast cancer patients with high expression of PBK/TOPK have a poor prognosis. Therefore, health care providers can optimize breast cancer management using this indicator.

\section{Background}

The incidence and mortality of breast cancer rank first among female malignant cancers in China and worldwide.[1, 2] The specific causes of breast cancer are still unclear; however, some high-risk groups such as individuals with family susceptibility, hormone disorders, or immune response system disorders are more likely to develop the disease.[3] Due to the diverse biological characteristics of breast cancer, studies on the pathogenesis and etiology are conducted based on molecular biology.[4]

Several critical signal transfer pathways play essential roles in breast cancer progression [4]; for example, the phosphoinositide 3-kinase/protein kinase B/mechanistic target of rapamycin (PI3K/AKT/mTOR) signaling pathway is often activated in breast cancer and plays an important role in the expansion and invasion of cancer cells.[5, 6] Human epidermal growth factor receptor 2 (HER-2) and estrogen receptor (ER)-a are upstream molecules of the PI3K/AKT/mTOR signaling pathway, which is very important for diagnosing and treating breast malignant tumors.[7] Hormone receptor-positive patients with breast cancer can be treated with endocrine therapy and have a good prognosis. On the contrary, hormone 
receptor-negative patients are not sensitive to endocrine therapy and have poor prognoses.[8] HER-2 is as an important predictor of breast cancer prognosis. It has been demonstrated that the over-expression of HER-2 indicates early metastasis and the rapid progression of tumors.[9] At present, clinically targeted drugs for HER-2 gene amplification are widely used clinically and have achieved definite curative effects by improving the prognosis of patients.[10] With in-depth study by researchers, new indicators that can be used as prognostic indicators for malignant tumors have been gradually uncovered.

PDZ-binding kinase/T lymphokine-activated killer cell-derived protein kinase (PBK/TOPK) is a serinethreonine protein kinase.[11] PBK/TOPK contains 322 amino acids and belongs to the family of mitogenactivated protein kinase molecules,[12] which are normally found in tissues with high proliferation potential, such as the testis and placenta, but not in normal tissues.[13-15] In recent years, studies have shown that PBK/TOPK is expressed in a variety of tumors, such as lung,[16] stomach,[17] and liver cancers. $[18,19]$ In addition, PBK/TOPK participates in many cell functions, including cell growth, DNA damage repair, the immune response, and inflammation,[20,21] and plays a vital role in cell cycle regulation and apoptosis.[22] Down-regulation of the PBK/TOPK gene can cause the internal structure of a cell to become disordered and leads to a delay in cell cytokinesis during mitosis, thereby reducing the cell viability and expansion capacity and inhibiting the potential for tumor cell generation.[23] It was reported that the over-expression of PBK/TOPK protein usually indicates a poor prognosis for malignant tumors.[15-18]

As a potential prognostic indicator for patients with breast cancer, PBK/TOPK has attracted increasing attention from clinicians and researchers. However, the clinical data on PBK/TOPK in breast cancer is still limited. Therefore, the objective of the present study was to explore the relationship between PBK/TOPK expression and clinicopathological indicators as well as the survival of patients with breast cancer. In addition, the influence of PBK/TOPK on the prognosis was evaluated to provide a foundation for clinical applications.

\section{Methods}

The present study was a retrospective analysis approved by the Ethics Committee of Zhongshan Hospital Affiliated to Dalian University (Ethical Review Number: 2019302). Due to the retrospective and anonymous nature of the study, informed consent from the patients was waived.

\section{Patients}

To collect long-term prognostic data, we screened patients diagnosed with breast cancer at the Department of Pathology from Zhongshan Hospital Affiliated with Dalian University from 1 January 2014 to 31 December 2014. The eligibility criteria for the patients were as follows: 1) patients pathologically diagnosed with invasive breast cancer; 2) no previous treatment for breast cancer; 3 ) the initial treatment after the diagnosis was surgery; 4) no radiotherapy, chemotherapy, or endocrine treatment was performed 
before the operation; 5) the postoperative specimens were available for further examination; 6) patients who completed follow-up; and 7) no history of malignancy.

\section{Data collection}

We collected the basic clinicopathological characteristics of the patients including the age, menopausal status, tumor size, TNM stage, histological grade, lymph node metastasis, number of lymph node metastases, vascular tumor thrombus, ER, progesterone receptor (PR), HER-2, and the nuclear protein Ki67. The exposure factor was the localization of PBK/TOPK protein in the nucleus or cytoplasm. The primary outcomes were overall survival (OS) and disease-free survival (DFS). OS was defined as the period from the day of the operation to the day of death for any reason or at the end of follow-up. The DFS was defined as the period from the day of the operation to the day of any disease progression (recurrence or metastasis) or the end of follow-up.

\section{Evaluation of the PBK/TOPK expression}

All paraffin tissue sections from the included patients were collected at Zhongshan Hospital Affiliated to Dalian University. PBK/TOPK antibody was purchased from Abcam (dilution 1:4000). Two pathologists with greater than 5 years of experience evaluated and scored the PBK/TOPK expression.

The standard procedures for preparing and evaluating PBK/TOPK expression were as follows: tissue sections were placed in a constant temperature oven at $60^{\circ} \mathrm{C}$ for $3 \mathrm{~h}$. After dewaxing and hydration, the antigen was repaired via placement in a microwave oven three times for $3 \mathrm{~min}$ with intervals of $5 \mathrm{~min}$ (3 min - 5 min - 3 min - 5 min - 3 min). Subsequent procedures consisted of incubation with a blocking solution of endogenous peroxidase at room temperature for $20 \mathrm{~min}$, incubation using a working solution of blocking goat serum at room temperature for the next 20 min, incubation with the PBK/TOPK antibody in a refrigerator at $4{ }^{\circ} \mathrm{C}$ overnight for $12 \mathrm{~h}$, incubation with biotin-labeled goat anti-rabbit IgG polymer at room temperature for $30 \mathrm{~min}$, incubation with horseradish enzyme-labeled streptomycin at room temperature for 30 min, color development using 3,3'-Diaminobenzidine (DAB) reagent after washing the slides with phosphate-buffered saline (PBS), and re-staining with hematoxylin for $15 \mathrm{~s}$. Finally, the slides were dehydrated, cleared, sealed, read, and recorded.

PBK/TOPK protein was localized in the nucleus or cytoplasm. Five fields of view for each patient were randomly selected under $400 x$ objective lens for scoring. First, the color of the rendering was scored (colorless ( 0 points), pale-yellow ( 1 point), brownish yellow ( 2 points), and brown ( 3 points)). Then, the color rendering ratio was calculated as follows: $1-10 \%$ for 1 point, $11-50 \%$ for 2 points, $51-80 \%$ for 3 points, and $>80 \%$ for 4 points. Next, the immunization score was generated by multiplying the above two scores. Furthermore, 0 points, 1-4 points, and $>4$ points were marked as '-,' '+,' and '++,', respectively (Figure 1). Finally, using 4 points as the cutoff value, we divided the expression of PBK/TOPK in breast cancer 
into two groups, with a score $\leq 4$ indicating low expression and a score $>4$ indicating high expression. [24]

\section{Statistical analysis}

SPSS 20.0 software was used to analyze the data in the present study. Categorical variables were described as counts and percentages while continuous variables were expressed as the mean and standard deviation (SD). The relationship between PBK/TOPK and clinicopathological parameters was evaluated with the Spearman's rank-order correlation. The difference in PBK/TOPK expression among different molecular types was evaluated with the chi square test. Kaplan-Meier analysis was used to generate survival curves and the log rank test was used to analyze OS and DFS. Prognostic correlation analysis was performed using univariate and multivariate Cox regression analyses. The receiver operating characteristic (ROC) curve was drawn to determine the sensitivity and specificity of PBK/TOPK for OS. All of the above analyses were performed with a threshold of $\mathrm{P}<0.05$ (two-sided) for statistical significance.

\section{Results}

\section{Characteristics of the included patients.}

We screened 863 patients with breast cancer treated in 2014 at our hospital. According to our eligibility criteria, 202 patients were included in the final analysis. The workflow for the screening processes is illustrated in Figure 2.

The mean age of the included patients was $52.41 \pm 10.97$ (mean \pm SD). Most of the tumors $(191 / 202$, $94.6 \%)$ were $\leq 5 \mathrm{~cm}$. Forty-one patients (20.3\%) had TNM stage III disease. The details of the baseline pathological characteristics are listed in Table 1.

\section{The expression of PBK/TOPK protein}

Among 202 breast cancer tissues, PBK/TOPK protein was expressed ('+' and '++') in 182 (90.1\%). According to our criteria, high expression and low expression of PBK/TOPK were found in 85 (42.1\%) and $117(57.9 \%)$ samples, respectively.

\section{Correlation between PBK/TOPK protein expression and clinicopathological characteristics}

According to the Spearman correlation analysis, histological grade $(P=0.015)$, TNM stages $(P=0.013)$, lymph node metastasis $(P=0.002)$, ER $(P=0.005), \mathrm{PR}(P=0.027)$, HER-2 $(P=0.037)$, and Ki-67 $(P<0.001)$ 
were associated with PBK/TOPK protein expression. However, the patients' age $(P=0.431)$, menopausal status ( $P=0.163)$, tumor size $(P=0.163)$, and vascular tumor thrombus $(P=0.110)$ had no significant correlation with PBK/TOPK protein expression. The detailed data for the correlation analysis are presented in Table 1.

In terms of the molecular types, high expression of PBK/TOPK was observed in $9.10 \%(3 / 33), 41.88 \%$ (31/74), 55.1\% (27/49), and 60.00\% (18/30) of patients with Luminal A-type, Luminal B type, HER-2 (+) type, and triple negative breast cancer (TNBC), respectively $(P<0.001$, Table 1$)$. According to the box plots (Figure 3), the median expression level of PBK/TOPK in Luminal A samples was lower than that in Luminal B samples. Additionally, the median expression levels of PBK/TOPK in Luminal A and Luminal B samples were lower than those of HER-2(+) and TNBC.

\section{PBK/TOPK protein expression level and prognosis}

The follow-up for all included patients ended on 31 December 2020 and 21 patients died due to breast cancer during this period. The median follow-up was 78 months (ranging from 72 to 83 months). The median OS and DFS were not calculated during the study period.

Kaplan-Meier analysis revealed that the expression level of PBK/TOPK was negatively correlated with the OS of breast cancer patients. The 5 -year survival rate was $81.2 \%$ and $95.7 \%$ for the high and low PBK/TOPK expression groups (Figure 4A). According to the Log-rank test, breast cancer patients with high PBK/TOPK expression had a poor prognosis $(P=0.001)$. Similarly, the expression level of PBK/TOPK was negatively correlated with the DFS of the patients. The 5 -year DFS rates were $78.8 \%$ and $92.3 \%$ for the high and low PBK/TOPK expression groups (Figure 4B). The Log-rank test showed that breast cancer patients with high PBK/TOPK expression had poor prognoses $(P=0.006)$.

Subgroup analysis was performed for OS according to the TNM stages using Kaplan-Meier analysis. For patients with stages I-II breast cancer, the expression level of PBK/TOPK was negatively correlated with OS. The 5-year OS rates were $86.7 \%$ and $95.8 \%$ for the high and low PBK/TOPK expression groups (Figure $4 C)$. According to the Log-rank test, breast cancer patients with high PBK/TOPK expression had poor prognoses $(P=0.042)$. For patients with stage III breast cancer, the expression level of PBK/TOPK was negatively correlated with OS as well. The 5 -year OS rates were $65.2 \%$ and $95.2 \%$ for the high and low PBK/TOPK expression groups (Figure 4D). According to the Log-rank test, breast cancer patients with high PBK/TOPK expression had poor prognoses $(P=0.018)$.

\section{Influential factors related to the prognosis}

Univariate Cox regression analysis revealed that tumor size (OS: hazard ratio $(H R)=4.148, P=0.010$; DFS: $\mathrm{HR}=3.452, P=0.007$ ), lymph node metastasis (OS: HR=3.390, $P=0.012$; DFS: HR=2.351, $P=0.032$ ), HER-2 (OS:HR=2.016, $P=0.002$; DFS:HR=1.977, $P=0.001$ ), and PBK/TOPK (OS: $\mathrm{HR}=4.590, P=0.003$; DFS: 
$\mathrm{HR}=2.890, P=0.009)$ were risk factors for both OS and DFS among the included patients. On the contrary, ER expression (OS: HR=0.342, $P=0.014$; DFS: $H R=0.401, P=0.018$ ) was a protective factor for the OS and DFS of the patients. Further, the TNM stage (OS: HR=2.658, $P=0.030$ ) was a risk factor for the OS of the patients. The results of the univariate analysis are listed in Table 2.

The meaningful indicators generated from the univariate analysis $(P<0.05)$ were included in the multivariate Cox regression, and the results are presented in Table 3 and Figure 5. High expression of PBK/TOPK was an independent influential factor on OS in breast cancer $(P=0.034)$ and HER-2 expression was an independent influential factor on DFS $(P=0.014)$. Analysis of the ROC curve in Figure 6. revealed that the area under the curve was 0.690 (95\% confidence interval (Cl): 0.576-0.805) for the expression of PBK/TOPK in the prediction of 5-year OS $(P=0.004)$.

\section{Discussion}

Breast cancer is a type of malignant tumor with high heterogeneity. Different molecular types of breast cancer have different clinical manifestations.[25] In this retrospective study, we explored PBK/TOPK protein expression in patients with breast cancer. PBK/TOPK protein is difficult to detect in normal tissues but has been found in a variety of malignant tumors.[15] Among 202 breast cancer samples, high expression of PBK/TOPK was found in 85 cases (42.1\%), consistent with a previous report (104/290, $35.9 \%)$ by O'Leary et al.[26]

In clinical practice, lymph node metastasis, the number of lymph nodes with metastasis, histological grade, TNM stage, ER, PR, HER-2, and Ki-67 are traditional prognostic indicators for patients with breast cancer.[27-30] In the present study, PBK/TOPK protein expression was positively associated with the risk factors for prognosis, including histological grade $(P=0.015)$, TNM stages $(P=0.013)$, lymph node metastasis $(P=0.002)$, HER-2 $(P=0.037)$, and Ki-67 $(P<0.001)$, but negatively associated with the beneficial factors $\operatorname{ER}(P=0.005)$ and $\mathrm{PR}(P=0.027)$. Furthermore, according to our survival analysis, the expression level of PBK/TOPK was negatively correlated with both the OS and the DFS of breast cancer patients. Using multivariate Cox regression analysis, we confirmed that PBK/TOPK was an independent risk factor for the prognosis of patients with breast cancer $(P=0.034)$. The finding that PBK/TOPK protein overexpression predicts poor prognosis for patients with breast cancer is consistent with a previous study [26] and other prognostic studies on other malignancies.[15-18, 31, 32]

In 2000, Perou et al. proposed a molecular classification system for breast cancer, comprising Luminal A, Luminal B, HER-2 overexpression, and basal-like type (including TNBC).[33] Luminal A has the best prognosis, whereas basal-like type breast cancer has the worst prognosis. Carey et al. followed 496 breast cancer patients for 8.1 to 11.2 years and concluded that the prognoses for patients with HER-2 overexpression and TNBC were the worst, whereas Luminal A had the best prognosis.[34] According to our analysis, the over-expression of PBK/TOPK in Luminal A was significantly lower than that of HER-2(+) and TNBC types, which once again confirmed that PBK/TOPK over-expression is associated with a worse prognosis for breast cancer. 
To the best of our knowledge, this study is the first investigation using clinical data to explore the expression of PBK/TOPK in breast cancer and its relationship with patient prognosis. We conducted several rigorous statistical tests, including correlation tests, Kaplan Meier curves, and Cox regression analysis, to confirm our findings. There are several limitations. First, the sample size for the study was relatively small. Additionally, not all specimens could be used in the study. Only slides stained immunohistochemically to detect the expression of PBK/TOPK protein in paraffin tissue samples of breast cancer were included in the final analysis. Next, due to the inherent characteristics of observational study, some potential bias cannot be avoided; for instance, 13 patients were lost to follow up, which can lead to missing information bias. Large-scale prospective studies are needed to confirm our findings in the future.

\section{Conclusion}

With regard to the practical implications, this study provides evidence that PBK/TOPK protein is overexpressed in breast cancer tissues and predicts a poor prognosis for patients with breast cancer, which can provide clinicians with diagnosis and treatment value.

PBK/TOPK protein is overexpressed in breast cancer and its expression is closely related to the clinicopathological characteristics of the disease. Breast cancer patients with high expression of PBK/TOPK have a poor prognosis. Therefore, health care providers can optimize breast cancer management using this indicator.

\section{Abbreviations}

PBK PDZ-binding-kinase

TOPK T-LAK cell-originated protein kinase

ER Estrogen Receptor

PR Progesterone Receptor

HER-2 Human epidermal growth factor receptor-2

Ki-67 Nuclear-associated antigen Ki-67

OS Overall Survival

DFS Disease free survival

PBS Phosphate buffer saline

DAB 3, 3N-Diaminobenzidine 


\section{Declarations}

Ethics approval and consent to participate: The present study was a retrospective analysis approved by the Ethics Committee of Zhongshan Hospital Affiliated to Dalian University (Ethical Review Number: 2019302). Due to the retrospective and anonymous nature of the study, informed consent from the patients was waived. All methods were performed in accordance with the relevant guidelines and regulations.

Consent for publication: Not applicable.

Availability of data and materials: The datasets used and/or analysed during the current study are available from the corresponding author on reasonable request.

Competing interests: The authors declare that they have no competing interests.

Funding: None.

Authors' contributions: All authors contributed to the study conception and design. Material preparation was performed by JX, YW, MZ, ZJ, and RZ. The experimental operation was done by LQ, JB, JY, ZG and SC. Data collection and analysis were performed by LQ and JB. The first draft of the manuscript was written by LQ and all authors commented on the previous versions of the manuscript. All authors read and approved the final version of the manuscript.

Acknowledgements: Editorial assistance was provided by Medjaden Inc.

\section{References}

1. Global, regional, and national burden of brain and other CNS cancer, 1990-2016: a systematic analysis for the Global Burden of Disease Study 2016. Lancet Neurol. 2019;18:376-93.

2. Jiang X, Tang H, Chen T. Epidemiology of gynecologic cancers in China. J Gynecol Oncol. 2018;29:e7.

3. Kamińska M, Ciszewski T, Łopacka-Szatan K, Miotła P, Starosławska E. Breast cancer risk factors. Przeglad menopauzalny = Menopause review. 2015;14:196-202.

4. Feng Y, Spezia M, Huang S, Yuan C, Zeng Z, Zhang L, et al. Breast cancer development and progression: Risk factors, cancer stem cells, signaling pathways, genomics, and molecular pathogenesis. Genes \& diseases. 2018;5:77-106.

5. Ortega MA, Fraile-Martínez O, Asúnsolo Á, Buján J, García-Honduvilla N, Coca S. Signal Transduction Pathways in Breast Cancer: The Important Role of PI3K/Akt/mTOR. J Oncol. 2020;2020:9258396.

6. Guerrero-Zotano A, Mayer IA, Arteaga CL. PI3K/AKT/mTOR: role in breast cancer progression, drug resistance, and treatment. Cancer Metastasis Rev. 2016;35:515-24. 
7. Araki K, Miyoshi Y. Mechanism of resistance to endocrine therapy in breast cancer: the important role of PI3K/Akt/mTOR in estrogen receptor-positive, HER2-negative breast cancer. Breast Cancer. 2018;25:392-401.

8. Cui X, Schiff R, Arpino G, Osborne CK, Lee AV. Biology of progesterone receptor loss in breast cancer and its implications for endocrine therapy. J Clin Oncol. 2005;23:7721-35.

9. Yarden Y. Biology of HER2 and its importance in breast cancer. Oncology. 2001;61 Suppl 2:1-13.

10. Slamon D, Eiermann W, Robert N, Pienkowski T, Martin M, Press M, et al. Adjuvant trastuzumab in HER2-positive breast cancer. N Engl J Med. 2011;365:1273-83.

11. Han Z, Li L, Huang Y, Zhao H, Luo Y. PBK/TOPK: A Therapeutic Target Worthy of Attention. Cells. $2021 ; 10$.

12. Matsumoto S, Abe Y, Fujibuchi T, Takeuchi T, Kito K, Ueda N, et al. Characterization of a MAPKK-like protein kinase TOPK. Biochem Biophys Res Commun. 2004;325:997-1004.

13. Gaudet S, Branton D, Lue RA. Characterization of PDZ-binding kinase, a mitotic kinase. Proc Natl Acad Sci U S A. 2000;97:5167-72.

14. Abe Y, Matsumoto S, Kito K, Ueda N. Cloning and expression of a novel MAPKK-like protein kinase, lymphokine-activated killer T-cell-originated protein kinase, specifically expressed in the testis and activated lymphoid cells. J Biol Chem. 2000;275:21525-31.

15. Zhang $Y$, Yang $X$, Wang R, Zhang X. Prognostic Value of PDZ-Binding Kinase/T-LAK Cell-Originated Protein Kinase (PBK/TOPK) in Patients with Cancer. J Cancer. 2019;10:131-7.

16. Lei B, Qi W, Zhao Y, Li Y, Liu S, Xu X, et al. PBK/TOPK expression correlates with mutant p53 and affects patients' prognosis and cell proliferation and viability in lung adenocarcinoma. Hum Pathol. 2015;46:217-24.

17. Ohashi T, Komatsu S, Ichikawa D, Miyamae M, Okajima W, Imamura T, et al. Overexpression of PBK/TOPK relates to tumour malignant potential and poor outcome of gastric carcinoma. $\mathrm{Br} J$ Cancer. 2017;116:218-26.

18. Yang QX, Zhong S, He L, Jia XJ, Tang H, Cheng ST, et al. PBK overexpression promotes metastasis of hepatocellular carcinoma via activating ETV4-uPAR signaling pathway. Cancer Lett. 2019;452:90102.

19. Cao H, Yang M, Yang Y, Fang J, Cui Y. PBK/TOPK promotes chemoresistance to oxaliplatin in hepatocellular carcinoma cells by regulating PTEN. Acta Biochim Biophys Sin (Shanghai). 2021;53:584-92.

20. Gao S, Zhu Y, Li H, Xia Z, Wu Q, Yao S, et al. Remote ischemic postconditioning protects against renal ischemia/reperfusion injury by activation of T-LAK-cell-originated protein kinase (TOPK)/PTEN/Akt signaling pathway mediated anti-oxidation and anti-inflammation. Int Immunopharmacol. 2016;38:395-401.

21. Ayllón V, O'Connor R. PBK/TOPK promotes tumour cell proliferation through p38 MAPK activity and regulation of the DNA damage response. Oncogene. 2007;26:3451-61. 
22. Shinde SR, Gangula NR, Kavela S, Pandey V, Maddika S. TOPK and PTEN participate in CHFR mediated mitotic checkpoint. Cell Signal. 2013;25:2511-7.

23. Lu S, Ye L, Yin S, Zhao C, Yan M, Liu X, et al. Glycyrol exerts potent therapeutic effect on lung cancer via directly inactivating T-LAK cell-originated protein kinase. Pharmacol Res. 2019;147:104366.

24. Tsuda $\mathrm{H}$. Individualization of breast cancer based on histopathological features and molecular alterations. Breast Cancer. 2008;15:121-32.

25. LoibI S, Poortmans P, Morrow M, Denkert C, Curigliano G. Breast cancer. Lancet. 2021;397:1750-69.

26. PC OL, Penny SA, Dolan RT, Kelly CM, Madden SF, Rexhepaj E, et al. Systematic antibody generation and validation via tissue microarray technology leading to identification of a novel protein prognostic panel in breast cancer. BMC Cancer. 2013;13:175.

27. Carter CL, Allen C, Henson DE. Relation of tumor size, lymph node status, and survival in 24,740 breast cancer cases. Cancer. 1989;63:181-7.

28. Cianfrocca M, Goldstein LJ. Prognostic and predictive factors in early-stage breast cancer. Oncologist. 2004;9:606-16.

29. Donegan WL. Tumor-related prognostic factors for breast cancer. CA Cancer J Clin. 1997;47:28-51.

30. Shapochka DO, Zaletok SP, Gnidyuk MI. Relationship between NF-kB, ER, PR, Her2/neu, Ki67, p53 expression in human breast cancer. Exp Oncol. 2012;34:358-63.

31. Ohashi T, Komatsu S, Ichikawa D, Miyamae M, Okajima W, Imamura T, et al. Overexpression of PBK/TOPK Contributes to Tumor Development and Poor Outcome of Esophageal Squamous Cell Carcinoma. Anticancer Res. 2016;36:6457-66.

32. Chang CF, Chen SL, Sung WW, Hsieh MJ, Hsu HT, Chen LH, et al. PBK/TOPK Expression Predicts Prognosis in Oral Cancer. Int J Mol Sci. 2016;17.

33. Perou CM, Sørlie T, Eisen MB, van de Rijn M, Jeffrey SS, Rees CA, et al. Molecular portraits of human breast tumours. Nature. 2000;406:747-52.

34. Carey LA, Perou CM, Livasy CA, Dressler LG, Cowan D, Conway K, et al. Race, breast cancer subtypes, and survival in the Carolina Breast Cancer Study. JAMA. 2006;295:2492-502.

\section{Tables}

Table 1. The clinicopathological characteristics of included patients and their correlations with the expression of PBK/TOPK 


\begin{tabular}{|c|c|c|c|c|c|}
\hline & Total, N (\%) & PBK/TOPK & & & \\
\hline Parameter & $(n=202)$ & Low, N (\%) & High, N (\%) & $r_{s}$ & $P$ \\
\hline \multicolumn{6}{|l|}{ Age } \\
\hline$\leq 50 y$ & $94(46.5)$ & $52(55.3)$ & $42(44.7)$ & -0.049 & 0.487 \\
\hline$>50 y$ & $108(53.5)$ & $65(60.2)$ & $43(39.8)$ & & \\
\hline \multicolumn{6}{|l|}{ Menopausal state } \\
\hline Premenopausal & 115 (56.9) & $63(54.8)$ & $52(45.2)$ & -0.073 & 0.301 \\
\hline Postmenopausal & $87(43.1)$ & $54(62.1)$ & $33(37.9)$ & & \\
\hline \multicolumn{6}{|l|}{ Tumor size } \\
\hline$\leq 2 \mathrm{~cm}$ & $95(47.0)$ & $61(64.2)$ & $34(35.8)$ & & \\
\hline$>2 \mathrm{~cm} \leq 5 \mathrm{~cm}$ & $96(47.6)$ & $51(53.1)$ & $45(46.9)$ & 0.124 & 0.078 \\
\hline$>5 \mathrm{~cm}$ & $11(5.4)$ & $5(45.5)$ & $6(54.5)$ & & \\
\hline \multicolumn{6}{|l|}{ Histological } \\
\hline G1 & $20(9.9)$ & $13(65.0)$ & $7(35.0)$ & & \\
\hline $\mathrm{G} 2$ & $147(72.8)$ & $93(63.3)$ & $54(36.7)$ & 0.171 & $0.015^{\star}$ \\
\hline G3 & 35 (17.3) & $11(31.4)$ & $24(68.6)$ & & \\
\hline \multicolumn{6}{|l|}{ TNM } \\
\hline 1 & $66(32.7)$ & $45(68.2)$ & $21(31.8)$ & & \\
\hline II & $95(47.0)$ & $54(56.8)$ & $41(43.2)$ & 0.175 & $0.013 *$ \\
\hline III & $41(20.3)$ & $18(43.9)$ & $23(56.1)$ & & \\
\hline \multicolumn{6}{|l|}{ Cancer thrombus } \\
\hline no & $172(85.1)$ & $103(59.9)$ & $69(40.1)$ & 0.095 & 0.178 \\
\hline yes & $30(14.9)$ & $14(46.7)$ & $16(53.3)$ & & \\
\hline \multicolumn{6}{|c|}{ Lymph node metastasis } \\
\hline no & $113(55.9)$ & $76(67.2)$ & $37(32.8)$ & 0.213 & $0.002^{*}$ \\
\hline yes & $89(44.1)$ & $41(46.1)$ & $48(53.9)$ & & \\
\hline \multicolumn{6}{|c|}{ Number of lymph nodes } \\
\hline $1-3$ & $56(27.7)$ & $29(51.8)$ & $27(48.2)$ & & \\
\hline $4-9$ & $20(9.9)$ & $10(50.0)$ & $10(50.0)$ & 0.241 & $0.001 *$ \\
\hline
\end{tabular}




\begin{tabular}{|llllll|}
\hline$\geq 10$ & $13(6.5)$ & $2(15.4)$ & $11(84.6)$ & & \\
\hline Estrogen receptor & & & & & \\
\hline Negative & $53(26.2)$ & $22(41.5)$ & $31(58.5)$ & -0.198 & $0.005^{\star}$ \\
\hline Positive & $149(73.8)$ & $95(63.8)$ & $54(36.2)$ & & \\
\hline Progesterone receptor & & & & & \\
\hline Negative & $110(54.5)$ & $56(50.9)$ & $54(49.1)$ & -0.155 & $0.027^{\star}$ \\
\hline Positive & $92(45.5)$ & $61(66.3)$ & $31(33.7)$ & & \\
\hline HER-2 & & & & & \\
\hline Negative & $137(67.8)$ & $85(62.0)$ & $52(38.0)$ & 0.153 & $0.037^{\star}$ \\
\hline Positive & $49(24.2)$ & $22(44.9)$ & $27(55.1)$ & & \\
\hline Ki-67 & & & & & \\
\hline Low expression & $65(32.2)$ & $52(80.0)$ & $13(20.0)$ & 0.312 & $<0.001^{\star}$ \\
\hline High expression & $137(67.8)$ & $64(46.7)$ & $73(53.3)$ & & \\
\hline Molecular typing & & & & & \\
\hline Luminal A & $33(16.3)$ & $30(90.9)$ & $3(9.1)$ & $22.031^{\wedge}$ & $<0.001^{\wedge}$ \\
\hline Luminal B & $74(36.6)$ & $43(58.1)$ & $31(41.9)$ & & \\
\hline HER-2(+) & $49(24.2)$ & $22(44.9)$ & $27(55.1)$ & & \\
\hline TNBC & $30(14.9)$ & $12(40.0)$ & $18(60.0)$ & & \\
\hline
\end{tabular}

* Spearman correlation statistical analysis. ${ }^{\wedge}$ Chi-square test.

Table 2. Univariate Cox regression analysis of OS and DFS for all included patients 


\begin{tabular}{|c|c|c|c|c|c|c|}
\hline \multirow[b]{2}{*}{ Variable } & \multicolumn{2}{|r|}{ os } & \multicolumn{4}{|c|}{ DFS } \\
\hline & HR & $95 \% \mathrm{Cl}$ & $P$ & HR & $95 \% \mathrm{Cl}$ & $P$ \\
\hline \multicolumn{7}{|l|}{ Age } \\
\hline ( $\leq 50$ y vs. $>50$ y) & 1.502 & $\begin{array}{l}0.623- \\
3.624\end{array}$ & 0.365 & 1.563 & $\begin{array}{l}0.716- \\
3.413\end{array}$ & 0.263 \\
\hline \multicolumn{7}{|l|}{ Menopausal state } \\
\hline $\begin{array}{l}\text { (Premenopausal vs } \\
\text { Postmenopausal) }\end{array}$ & 0.781 & $\begin{array}{l}0.342- \\
1.884\end{array}$ & 0.582 & 0.887 & $\begin{array}{l}0.412- \\
1.912\end{array}$ & 0.760 \\
\hline \multicolumn{7}{|l|}{ Tumor size } \\
\hline$(\leq 2 \mathrm{~cm}$ vs. $>2 \mathrm{~cm})$ & 4.148 & $\begin{array}{l}1.395- \\
12.330\end{array}$ & $0.010^{\star}$ & 3.452 & $\begin{array}{l}1.393- \\
8.557\end{array}$ & $0.007 *$ \\
\hline \multicolumn{7}{|l|}{ Histological grade } \\
\hline (G1, G2, or G3) & 2.020 & $\begin{array}{l}0.784- \\
5.208\end{array}$ & 0.145 & 1.812 & $\begin{array}{l}0.766- \\
4.285\end{array}$ & 0.176 \\
\hline \multicolumn{7}{|l|}{ TNM stage } \\
\hline (I, II, vs III) & 2.658 & $\begin{array}{l}1.101- \\
6.414\end{array}$ & $0.030^{*}$ & 2.195 & $\begin{array}{l}0.986- \\
4.887\end{array}$ & 0.054 \\
\hline \multicolumn{7}{|l|}{ Cancer thrombus } \\
\hline (No vs yes) & 2.505 & $\begin{array}{l}0.972- \\
6.457\end{array}$ & 0.057 & 1.829 & $\begin{array}{l}0.738- \\
4.533\end{array}$ & 0.192 \\
\hline \multicolumn{7}{|l|}{ Lymph node metastasis } \\
\hline (No vs yes) & 3.390 & $\begin{array}{l}1.315- \\
8.742\end{array}$ & $0.012^{\star}$ & 2.351 & $\begin{array}{l}1.076- \\
5.136\end{array}$ & $0.032 *$ \\
\hline \multicolumn{7}{|l|}{ Estrogen receptor } \\
\hline (Negative vs Positive) & 0.342 & $\begin{array}{l}0.145- \\
0.806\end{array}$ & $0.014^{*}$ & 0.401 & $\begin{array}{l}0.187- \\
0.856\end{array}$ & $0.018 *$ \\
\hline \multicolumn{7}{|c|}{$\begin{array}{l}\text { Progesterone receptor (Negative vs. } \\
\text { Positive) }\end{array}$} \\
\hline & 0.443 & $\begin{array}{l}0.172- \\
0.142\end{array}$ & 0.092 & 0.671 & $\begin{array}{l}0.307- \\
1.465\end{array}$ & 0.317 \\
\hline \multicolumn{7}{|l|}{ HER-2 } \\
\hline (Negative vs Positive) & 2.016 & $\begin{array}{l}1.283- \\
3.168\end{array}$ & $0.002^{*}$ & 1.977 & $\begin{array}{l}1.328- \\
2.943\end{array}$ & $0.001 *$ \\
\hline \multicolumn{7}{|l|}{ Ki-67 } \\
\hline (Low vs High) & 2.105 & $\begin{array}{l}0.708- \\
6.257\end{array}$ & 0.180 & 2.146 & $\begin{array}{l}0.812- \\
5.666\end{array}$ & 0.123 \\
\hline
\end{tabular}




\section{PBK/TOPK}

(Low vs High)

$4.590 \quad 1.681-$

$0.003 * \quad 2.890$

$1.298-$

6.433

0.009*

\section{Chemotherapy}

(Yes vs. No)

$\begin{array}{ll}2.003 \quad 0.268- \\ & 15.018\end{array}$

0.498

2.669

$0.361-$

19.728

0.336

*Cox ratio univariate analysis is statistically significant $(P<0.05)$.

Table 3. Multivariate Cox regression analysis of OS and DFS for all included patients

\begin{tabular}{|c|c|c|c|c|c|c|}
\hline \multirow[b]{2}{*}{ Variable } & \multicolumn{2}{|r|}{ OS } & \multicolumn{4}{|c|}{ DFS } \\
\hline & HR & $95 \% \mathrm{Cl}$ & $P$ & HR & $95 \% \mathrm{Cl}$ & $P$ \\
\hline \multicolumn{7}{|l|}{ TNM } \\
\hline$(\mathrm{I}, \mathrm{II}$, or III) & 1.002 & $0.355-2.829$ & 0.997 & -- & -- & -- \\
\hline \multicolumn{7}{|l|}{ Lymph node metastasis } \\
\hline (No vs yes) & 2.139 & $0.714-6.408$ & 0.174 & 1.574 & $0.705-3.513$ & 0.268 \\
\hline \multicolumn{7}{|l|}{ Tumor size } \\
\hline$(\leq 2 \mathrm{~cm}$ vs $>2 \mathrm{~cm})$ & 2.636 & $0.865-8.026$ & 0.088 & 2.461 & $0.975-6.212$ & 0.057 \\
\hline \multicolumn{7}{|l|}{ ER } \\
\hline (Negative vs Positive) & 0.572 & $0.236-1.388$ & 0.217 & 0.636 & $0.289-1.398$ & 0.260 \\
\hline \multicolumn{7}{|l|}{ HER-2 } \\
\hline (Negative vs Positive) & 1.566 & $0.996-2.464$ & 0.052 & 1.633 & $1.104-2.416$ & $0.014 *$ \\
\hline \multicolumn{7}{|l|}{ PBK/TOPK } \\
\hline (Low vs High) & 3.065 & $1.091-8.610$ & $0.034^{\star}$ & 2.091 & $0.918-4.764$ & 0.079 \\
\hline
\end{tabular}

${ }^{*}$ Cox ratio multivariate analysis is statistically significant $(P<0.05)$

\section{Figures}

\section{Figure 1}


PBK/TOPK protein expression in breast cancer tissues (original magnification $\times 400$ ). A) High expression of PBK/TOPK; B) Low expression of PBK/TOPK; C) No expression of PBK/TOPK.

\section{Figure 2}

Flow chart of the study screening process.

\section{Figure 3}

Box plot of PBK/TOPK expression in molecular typing of breast cancer.

\section{Figure 4}

Kaplan-Meier curve according to PBK/TOPK expression. A) For OS. B) For DFS, C) For OS in TNM stages I-II breast cancer patients. D) For OS in TNM stage III breast cancer patients.

\section{Figure 5}

Forest plot for multivariate Cox regression analysis of OS and DFS for all included patients.

\section{Figure 6}

The ROC curve of PBK/TOPK overexpression for prediction of overall survival. 